\title{
Non-quadratic local stabilization for continuous-time
}

\section{Takagi-Sugeno models}

\author{
Thierry Marie Guerra ${ }^{(1)}$, Miguel Bernal ${ }^{(2)}$, Kevin Guelton ${ }^{(3), *}$, Salim Labiod ${ }^{(4)}$ \\ ${ }^{(1)}$ LAMIH FRE CNRS 3304, \\ University of Valenciennes Hainaut-Cambrésis, France \\ phone: +33 3275114 87; email: guerra@univ-valenciennes.fr \\ ${ }^{(2)}$ Sonora Institute of Technology \\ 5 de febrero 818 Sur, Col. Centro, C.P. 85000. Ciudad Obregón, Mexico. \\ email: miguel.bernal@itson.edu.mx \\ *Corresponding author \\ ${ }^{(3)}$ CReSTIC EA 3804, \\ University of Reims Champagne-Ardenne, \\ Moulin de la Housse, BP 1039, 51687 Reims cedex 2, France. \\ Phone : +33 326913261 ; email : kevin.guelton@univ-reims.fr \\ (4) University of Jijel, \\ LAMEL, \\ BP 98, Ouled Aissa Jijel 18000, Algérie.
}

\section{Abstract :}

This paper is concerned with non-quadratic stabilization of continuous-time Takagi-Sugeno (TS) models. The well-known problem of handling time-derivatives of membership functions (MFs) as to obtain conditions in the form of linear matrix inequalities (LMIs) is overcome by reducing global goals to the estimation of a region of attraction. Instead of parallel distributed compensation (PDC), a non-PDC control law is proposed according to the non-quadratic nature of the Lyapunov function. Examples are provided to show the advantages over the quadratic and some non-quadratic approaches.

\section{Keywords :}

Linear Matrix Inequalities, Non-quadratic Lyapunov function, Stabilization, Takagi-Sugeno models. 


\section{Introduction}

Since they were proposed in [1] the control community has intensively studied Takagi-Sugeno (TS) models due to the fact that they can exactly represent a nonlinear model in a compact subset of the domain of the state variables. A TS model is a nonlinear blending of linear models via membership functions (MFs) which hold the convex-sum property [2]. The stabilization problem is usually addressed via the so-called parallel distributed compensation (PDC) control law [3], which is a nonlinear blending of linear-state feedbacks which uses the same MFs as the TS model.

The direct Lyapunov method altogether with quadratic Lyapunov functions has been usually employed to investigate the stability and stabilization of TS models. This method usually leads to conditions formulated in terms of linear matrix inequalities (LMIs) [4], which can be efficiently solved by convex optimization techniques. Quadratic analysis and design has produced a remarkable number of results regarding robustness, performance, observer design, output feedback and time delay systems (see e.g. [5-9] and references therein). Nonetheless, the quadratic approach presents serious limitations because its solutions are inherently pessimistic, i.e., there are stable or stabilizable models which do not have a quadratic solution (see [10] and references therein). Conservativeness comes from different sources: the type of TS model [11,12], the way the membership functions are dropped-off to obtain LMI expressions [13-15], the integration of membership-function information $[16,17]$, or the choice of Lyapunov function $[18,19]$. This work is concerned with a relaxation in the latter sense which demands a change of perspective from global to local conditions.

Several Lyapunov functions have been proposed in the literature. Piecewise Lyapunov functions (PWLF) $[18,20]$ have been straightforwardly applied to those TS models that induce state-space partitions from the fact that not all their linear components are simultaneously activated; unfortunately, TS models constructed via the sector nonlinearity approach lack this property. Various kinds of non-quadratic Lyapunov functions 
(NQLF) have been also employed; they depend on the same MFs of the model, hereby taking into account structural information otherwise ignored by the quadratic approach. However, NQLF-based results have not triggered the same developments for continuous-time TS models [21] than for discrete-time ones [22,26]. This asymmetry is explained by the difficulty of dealing with time-derivatives of the MFs that emerge while applying the direct Lyapunov method to obtain global conditions.

Some palliative solutions have been proposed to the aforementioned problem. In $[21,27]$ the authors bound the time-derivatives of the MFs assuming that they do not depend on the input, which turns out to be very restrictive. Moreover, the proposed control law makes use of the time-derivatives of the MFs through a classical PDC scheme, thus ignoring the non-quadratic nature of the involved Lyapunov function. In [28] a line-integral Lyapunov function is proposed to circumvent the MFs' time-derivative obstacle, though the line integral is asked to be path-independent thus significantly reducing its applicability [29]. A change of perspective for non-quadratic stability analysis of TS models has been proposed in [30]. This approach reduces global goals to less exigent conditions, thereby showing that an estimation of the region of attraction can be found (local stability); this solution parallelizes nonlinear analysis and design for models that do not admit a global solution [31]. Said in another words, classical linear parameter varying (LPV) models do not capture the nonlinear behavior of models [32] whereas TS ones, also known as quasi-LPV models, can do it exactly [33]. In the LPV case it is therefore reasonable to deal with global stability (under the constraints due to the bounds on the variables) [32], whereas obviously global stability is very often unreachable for nonlinear systems and therefore for their TS representation. This paper extends the previous results so they can be applied for controller design. A non-PDC control law is employed to fully exploit the non-quadratic properties of the Lyapunov function.

The contents are organized as follows: Section II introduces TS models, sector nonlinearity approach, the NQLF this work is based on and the proposed non-PDC control law; a problem statement is made. Section III summarizes the work presented in [30] and extends it for stabilization purposes, thus establishing new 
local conditions for controller design. Section IV presents some illustrative examples to stress the fact that the few existent solutions on the subject are clearly outperformed by the new approach. Finally, section V gathers some conclusions and perspectives.

\section{Definitions and Notation}

Consider a nonlinear model of the form:

$\dot{x}(t)=f(z(t)) x(t)+g(z(t)) u(t)$

with $f(\cdot), g(\cdot)$ nonlinear functions, $x(t) \in \square^{n}$ the state vector, $u(t) \in \square^{m}$ the input vector, and $z(x(t)) \in \square^{p}$ the premise vector assumed to be bounded and smooth in a compact set $C$ of the state space including the origin.

Let $n l_{j}(t) \in\left[\underline{n l}_{j}, \overline{n l_{j}}\right], j \in\{1, \cdots, p\}$ be the set of bounded nonlinearities in (1) belonging to $C$. Employing the sector nonlinearity approach [5], the following weighting functions can be constructed

$w_{0}^{j}(\cdot)=\frac{\overline{n l_{j}}-n l_{j}(\cdot)}{\overline{n l_{j}}-\underline{n l_{j}}}, \quad w_{1}^{j}(\cdot)=1-w_{0}^{j}(\cdot), j \in\{1, \cdots, p\}$.

From the previous weights, the following MFs are defined:

$h_{i}=h_{1+i_{1}+i_{2} \times 2+\cdots+i_{p} \times 2^{p-1}}=\prod_{j=1}^{p} w_{i_{j}}^{j}\left(z_{j}\right)$

with $i \in\left\{1, \cdots, 2^{p}\right\}, i_{j} \in\{0,1\}$. These MFs satisfy the convex sum property $\sum_{i=1}^{r} h_{i}(\cdot)=1, h_{i}(\cdot) \geq 0$ in $C$.

Where convenient, convex sums will be denoted as $\Upsilon_{z}=\sum_{i=1}^{r} h_{i}(z(t)) \Upsilon_{i}$ and their inverse as 


$$
\Upsilon_{z}^{-1}=\left(\sum_{i=1}^{r} h_{i}(z(t)) \Upsilon_{i}\right)^{-1}
$$

Based on the previous definitions, an exact representation of (1) in $C$ is given by the following TS model:

$$
\dot{x}(t)=\sum_{i=1}^{r} h_{i}(z(t))\left(A_{i} x(t)+B_{i} u(t)\right)=A_{z} x(t)+B_{z} u(t)
$$

with $r=2^{p} \in \square$ representing the number of linear models and $\left(A_{i}, B_{i}\right), i=1, \ldots, r$ a set of controllable pairs of proper dimensions directly obtained from the new representation.

Instead of PDC [3], the following generalization of the non-quadratic control law in [22] is proposed:

$u(t)=\sum_{i=1}^{r} \sum_{j=1}^{r} h_{i} h_{j}\left(F_{i}+\sum_{k=1}^{p} \frac{\partial w_{0}^{k}}{\partial z_{k}}\left(\frac{\partial z_{k}(t)^{T}}{\partial x(t)} A_{j} x\right) \cdot G_{i}^{k}\right)\left(\sum_{l=1}^{r} h_{l} P_{l}\right)^{-1} x(t)=\left(F_{z}+\delta(z)\right) P_{z}^{-1} x(t)$

with $F_{i}, G_{i}^{k} \in \square^{m \times n}$ the controller gains, $P_{i} \in \square^{n \times n}, P_{i}=P_{i}^{T}>0$, and $\delta(z)=\sum_{k=1}^{p} \frac{\partial w_{0}^{k}}{\partial z_{k}}\left(\frac{\partial z_{k}(t)^{T}}{\partial x(t)} A_{z} x\right) \cdot G_{z}^{k}$.

The closed-loop TS model is thence written as

$\dot{x}(t)=\sum_{i=1}^{r} h_{i}(z(t))\left(A_{i} x(t)+B_{i} u(t)\right)=\left(A_{z}+B_{z}\left(F_{z}+\delta(z)\right) P_{z}^{-1}\right) x(t)$.

Triple convex sums $\Upsilon_{z z z}=\sum_{i=1}^{r} \sum_{j=1}^{r} \sum_{l=1}^{r} h_{i}(z(t)) h_{j}(z(t)) h_{l}(z(t)) \Upsilon_{i j l}$ arise along matrix manipulations and their negative-definiteness is usually examined after dropping the MFs to obtain LMI conditions in terms of $\Upsilon_{i j l}$. The way the MFs are dropped from the triple sum above is called a sum-relaxation; several of them are available in the literature for double [13] and multiple sums [14]. In this work, a relaxation derived from Proposition 2 of the latter work has been adopted because it combines computational efficiency and does 
not need slack variables:

Relaxation Lemma [14]: Let $\Upsilon_{i j l}$ be matrices of proper dimensions. Then

$\sum_{i=1}^{r} \sum_{j=1}^{r} \sum_{l=1}^{r} h_{i}(z(t)) h_{j}(z(t)) h_{l}(z(t)) \Upsilon_{i j l}<0$

holds if for a given $q \geq 3$

$\tilde{\Upsilon}_{\mathbf{a}}=\sum_{\substack{\mathbf{b} \in \mathbf{A}(\mathbf{a}) \\ i j l=b_{1} b_{2} b_{3}}} \Upsilon_{i j l}<0, \quad \forall \mathbf{a} \in \mathbf{I}_{q}^{+}$

with $\mathbf{I}_{q}=\left\{\mathbf{a}=\left(a_{1}, \ldots, a_{q}\right) \in \square^{q}: 1 \leq a_{b} \leq r, \forall b=1, \ldots, q\right\}, \mathbf{I}_{q}^{+}=\left\{\mathbf{a} \in \mathbf{I}_{q}: a_{b} \leq a_{b+1}, \forall b=1, \ldots, q-1\right\}$, and $\mathbf{A}(\mathbf{a})$

being the set of permutations (with possibly repeated elements) of multi-index a in $\mathbf{I}_{q}$.

Quadratic Lyapunov function $V=x(t)^{T} P x(t)$ has been originally employed for stabilization purposes. TS model (4) is stabilized by the PDC control law $u(t)=\sum_{i=1}^{r} h_{i}(z(t)) F_{i} x(t)$ if there exist matrices $X=P^{-1}>0$ and $M_{i}=F_{i} X, i \in\{1, \cdots, r\}$ such that (8) holds with $\Upsilon_{i j}=X A_{i}^{T}+A_{i} X+M_{j}^{T} B_{i}^{T}+B_{i} M_{j}$ [5]. This result presents a serious inconvenient: as the number of rules increases the existence of a matrix $P$ as a common solution of the aforementioned set of LMIs gets harder to satisfy. In other words, asking for a global common Lyapunov function is a source of conservatism which can be reduced considering non-quadratic Lyapunov function (NQLF) candidates. NQLFs increase the flexibility by enlarging the choice of matrices that solve the LMI problem (8) $[19,22,27]$. In this framework, let us consider the following non-quadratic Lyapunov function candidate:

$V(x(t))=x(t)^{T}\left(\sum_{i=1}^{r} h_{i}(z(t)) P_{i}\right)^{-1} x(t)=x(t)^{T} P_{z}^{-1} x(t)$ 
with $P_{z}=P_{z}^{T}>0$ (therefore $\left.P_{z}^{-1}>0\right)$.

Its time-derivative along the trajectories of TS model (6) is given by

$$
\dot{V}(x(t))=x^{T}(t)\left(P_{z}^{-1}\left(A_{z}+B_{z}\left(F_{z}+\delta(z)\right) P_{z}^{-1}\right)+\left(A_{z}+B_{z}\left(F_{z}+\delta(z)\right) P_{z}^{-1}\right)^{T} P_{z}^{-1}+\dot{P}_{z}^{-1}\right) x(t) .
$$

Via elementary properties and taking into account that $P_{z} \dot{P}_{z}^{-1} P_{z}=-\dot{P}_{z}$, it is verified that

$$
\begin{aligned}
& \dot{V}(x(t))<0 \Leftrightarrow P_{z}^{-1}\left(A_{z}+B_{z}\left(F_{z}+\delta(z)\right) P_{z}^{-1}\right)+\left(A_{z}+B_{z}\left(F_{z}+\delta(z)\right) P_{z}^{-1}\right)^{T} P_{z}^{-1}+\dot{P}_{z}^{-1}<0 \\
& \quad \Leftrightarrow A_{z} P_{z}+B_{z}\left(F_{z}+\delta(z)\right)+\left(A_{z} P_{z}+B_{z}\left(F_{z}+\delta(z)\right)\right)^{T}+P_{z} \dot{P}_{z}^{-1} P_{z}<0 \\
& \Leftrightarrow A_{z} P_{z}+B_{z}\left(F_{z}+\delta(z)\right)+\left(A_{z} P_{z}+B_{z}\left(F_{z}+\delta(z)\right)\right)^{T}-\dot{P}_{z}<0
\end{aligned}
$$

Global conditions in the form of LMIs are normally derived from expressions similar to the previous one in the quadratic case. Unfortunately, obtaining non-conservative LMIs from (11) for global stabilization is no longer possible since the terms $\dot{P}_{z}=\sum_{i=1}^{r} \dot{h}_{i} P_{i}$ and $\delta(z)=\sum_{k=1}^{p} \frac{\partial w_{0}^{k}}{\partial z_{k}}\left(\frac{\partial z_{k}(t)^{T}}{\partial x(t)} A_{z} x\right) \cdot G_{z}^{k}$ depend on the timederivatives of the MFs, which do not convey to readily available bounds. This situation raises some questions:

- Should the quadratic case fail, what can be done?

- Can expression (11) be handled to avoid conditions of the sort $\left\|\dot{P}_{z}\right\|<\phi_{z}[19,21,27]$ ?

The previous works intended to derive global asymptotic conditions. In contrast, in [30] the previous questions were answered for stability analysis via a local approach. It was shown that reducing global goals responds better to stability problems, since the stability domain of a TS model can be estimated via local asymptotic conditions. This kind of estimation is customary for numerous nonlinear models whose global stability cannot be reached [31] and will be extended to the closed-loop TS model in the following section. 


\section{Main results}

Theorem 1 (Local stabilizability): If there exist matrices of the proper size $P_{i}=P_{i}^{T}>0, F_{i}$, and $G_{i}^{k}$, $i \in\{1, \ldots, r\}, k \in\{1, \ldots, p\}$ such that $A_{z} P_{z}+B_{z}\left(F_{z}+\delta(z)\right)+\left(A_{z} P_{z}+B_{z}\left(F_{z}+\delta(z)\right)\right)^{T}<0$, then there exists a domain $D, 0 \in D$, such that TS model (4) is locally asymptotically stabilizable under control law (5).

Proof: The NQLF candidate (9) satisfies $V(0)=0$ and $V(x) \geq 0$ in $\square^{n}$. Its time-derivative (10) holds $\dot{V}(0)=0$. Provided that $A_{z} P_{z}+B_{z}\left(F_{z}+\delta(z)\right)+\left(A_{z} P_{z}+B_{z}\left(F_{z}+\delta(z)\right)\right)^{T}<0$ it is implied that there exists a sufficiently small $\lambda>0$ such that $A_{z} P_{z}+B_{z}\left(F_{z}+\delta(z)\right)+\left(A_{z} P_{z}+B_{z}\left(F_{z}+\delta(z)\right)\right)^{T}+\lambda I<0$ which can be used to define $D=\left\{x: x \in B,\left\|\dot{P}_{z}\right\|<\lambda\right\}$. The origin belongs to domain $D$ since $\dot{P}_{z}=\sum_{i=1}^{r} \dot{h}_{i} P_{i}=\sum_{i=1}^{r}\left(\frac{\partial h_{i}}{\partial z}\right)^{T} \frac{\partial z^{T}}{\partial x} \dot{x} P_{i}=\sum_{i=1}^{r}\left[\left(\frac{\partial h_{i}}{\partial z}\right)^{T} \frac{\partial z^{T}}{\partial x}\left(A_{z}+B_{z}\left(F_{z}+\delta(z)\right) P_{z}^{-1}\right) x(t)\right] P_{i}$ depends on the state vector $x(t)$ in such a way that $x(t)=0$ is a trivial solution of $\left\|\dot{P}_{z}\right\|<\lambda$. Since $V(x)>0$ and $\dot{V}(x)<0$ in $D-\{0\}$, the equilibrium point $x=0$ is locally asymptotically stable, thus concluding the proof.

In [30] it has been shown that $\dot{P}_{z}$ can be written as

$\dot{P}_{z}=\sum_{k=1}^{p} \frac{\partial w_{0}^{k}}{\partial z_{k}}\left(P_{g_{1}(z, k)}-P_{g_{2}(z, k)}\right) \dot{z}_{k}=\sum_{j=1}^{r} \sum_{k=1}^{p} h_{j} \frac{\partial w_{0}^{k}}{\partial z_{k}}\left(P_{g_{1}(j, k)}-P_{g_{2}(j, k)}\right) \dot{z}_{k}$

with $g_{1}(j, k)=\left\lfloor(j-1) / 2^{p+1-k}\right\rfloor \times 2^{p+1-k}+1+(j-1) \bmod 2^{p-k}$ and $g_{2}(j, k)=g_{1}(j, k)+2^{p-k}$.

Substituting (12) in (11) and recalling that $\quad \dot{z}_{k}=\frac{\partial z_{k}(t)^{T}}{\partial x(t)}\left(A_{z} x(t)+B_{z} u(t)\right) \quad$ and 


$$
\begin{aligned}
& \delta(z)=\sum_{k=1}^{p} \frac{\partial w_{0}^{k}}{\partial z_{k}} \cdot\left(\frac{\partial z_{k}(t)^{T}}{\partial x(t)} A_{z} x\right) \cdot G_{z}^{k}, \text { gives } \\
& A_{z} P_{z}+B_{z}\left(F_{z}+\delta(z)\right)+\left(A_{z} P_{z}+B_{z}\left(F_{z}+\delta(z)\right)\right)^{T}-\sum_{k=1}^{p} \frac{\partial w_{0}^{k}}{\partial z_{k}}\left(P_{g_{1}(z, k)}-P_{g_{2}(z, k)}\right) \dot{z}_{k} \\
& =A_{z} P_{z}+P_{z} A_{z}^{T}+B_{z} F_{z}+F_{z}^{T} B_{z}^{T}+B_{z} \delta(z)+\delta^{T}(z) B_{z}^{T}-\sum_{k=1}^{p} \frac{\partial w_{0}^{k}}{\partial z_{k}} \cdot \frac{\partial z_{k}(t)^{T}}{\partial x(t)}\left(A_{z} x(t)+B_{z} u(t)\right)\left(P_{g_{1}(z, k)}-P_{g_{2}(z, k)}\right) \\
& =A_{z} P_{z}+B_{z} F_{z}+P_{z} A_{z}^{T}+F_{z}^{T} B_{z}^{T}-\sum_{k=1}^{p} \frac{\partial w_{0}^{k}}{\partial z_{k}} \cdot \frac{\partial z_{k}(t)^{T}}{\partial x(t)} B_{z} u(t)\left(P_{g_{1}(z, k)}-P_{g_{2}(z, k)}\right) \\
& -\sum_{k=1}^{p} \frac{\partial w_{0}^{k}}{\partial z_{k}} \cdot \frac{\partial z_{k}(t)^{T}}{\partial x(t)} A_{z} x(t)\left(P_{g_{1}(z, k)}-P_{g_{2}(z, k)}\right)+\sum_{k=1}^{p} \frac{\partial w_{0}^{k}}{\partial z_{k}} \cdot \frac{\partial z_{k}(t)^{T}}{\partial x(t)} A_{z} x\left(B_{z} G_{z}^{k}+\left(B_{z} G_{z}^{k}\right)^{T}\right) \\
& =A_{z} P_{z}+B_{z} F_{z}+P_{z} A_{z}^{T}+F_{z}^{T} B_{z}^{T}-\sum_{k=1}^{p} \frac{\partial w_{0}^{k}}{\partial z_{k}} \cdot \frac{\partial z_{k}(t)^{T}}{\partial x(t)} B_{z} u(t)\left(P_{g_{1}(z, k)}-P_{g_{2}(z, k)}\right) \\
& -\sum_{k=1}^{p} \frac{\partial w_{0}^{k}}{\partial z_{k}} \cdot \frac{\partial z_{k}(t)^{T}}{\partial x(t)} A_{z} x(t)\left(P_{g_{1}(z, k)}-P_{g_{2}(z, k)}-B_{z} G_{z}^{k}-\left(G_{z}^{k}\right)^{T} B_{z}^{T}\right) \\
& =A_{z} P_{z}+B_{z} F_{z}+P_{z} A_{z}^{T}+F_{z}^{T} B_{z}^{T}-\sum_{k=1}^{p} \sum_{v=1}^{n} \sum_{e=1}^{m} \frac{\partial w_{0}^{k}}{\partial x_{v}} u_{e}\left(B_{z}\right)_{v e}\left(P_{g_{1}(z, k)}-P_{g_{2}(z, k)}\right) \\
& -\sum_{k=1}^{p} \sum_{v=1}^{n} \sum_{s=1}^{n}\left(A_{z}\right)_{v s} \frac{\partial w_{0}^{k}}{\partial x_{v}} x_{s}\left(P_{g_{1}(z, k)}-P_{g_{2}(z, k)}-B_{z} G_{z}^{k}-\left(G_{z}^{k}\right)^{T} B_{z}^{T}\right)<0
\end{aligned}
$$

Therefore, knowing the bounds $\left|\frac{\partial w_{0}^{k}}{\partial x_{v}} x_{s}\right|$ and $\left|\frac{\partial w_{0}^{k}}{\partial x_{v}} u_{e}\right|$ allows (13) to be verified as an LMI constraint, thence guaranteeing $\dot{V}(x(t))<0$ in the region thus defined.

To guarantee $\|u\|_{2}<\mu$ (so as to bound $\left|\frac{\partial w_{0}^{k}}{\partial x_{v}} u_{e}\right|$ ), let us assume $|x(0)|<c$. Note that $P_{z} \geq c^{2} I$ (or $\left.P_{z}^{-1} \leq c^{-2} I\right)$ altogether with the fact that $x^{T}(t) P_{z}^{-1} x(t) \leq x^{T}(0) P_{z}^{-1} x(0)$ for $t \geq 0$, means that $V(x(t)) \leq 1$; then, condition $\|u\|_{2} \leq \mu$ is implied by 
$\frac{1}{\mu^{2}}\|u\|_{2}^{2}=\frac{1}{\mu^{2}} x^{T}(t) P_{z}^{-1}\left(F_{z}+\delta(z)\right)^{T}\left(F_{z}+\delta(z)\right) P_{z}^{-1} x(t) \leq x^{T}(t) P_{z}^{-1} x(t) \leq x^{T}(0) P_{z}^{-1} x(0) \leq 1$,

from which the following stems using the Schur complement and replacing $\delta(z)$ by its value

$$
\begin{aligned}
& x^{T}(t)\left(\frac{1}{\mu^{2}} P_{z}^{-1}\left(F_{z}+\delta(z)\right)^{T}\left(F_{z}+\delta(z)\right) P_{z}^{-1}-P_{z}^{-1}\right) x(t) \leq 0 \\
& \Leftrightarrow \frac{1}{\mu^{2}}\left(F_{z}+\delta(z)\right)^{T}\left(F_{z}+\delta(z)\right)-P_{z} \leq 0 \\
& \Leftrightarrow\left[\begin{array}{cc}
P_{z} & \left(F_{z}+\delta(z)\right)^{T} \\
F_{z}+\delta(z) & \mu^{2} I_{m}
\end{array}\right]>0 \Leftrightarrow\left[\begin{array}{cc}
P_{z} & F_{z}^{T} \\
F_{z} & \mu^{2} I_{m}
\end{array}\right]+\left[\begin{array}{cc}
0 & \delta^{T}(z) \\
\delta(z) & 0
\end{array}\right]>0 \\
& \Leftrightarrow\left[\begin{array}{cc}
P_{z} & F_{z}^{T} \\
F_{z} & \mu^{2} I_{m}
\end{array}\right]+\sum_{k=1}^{p} \frac{\partial w_{0}^{k}}{\partial z_{k}} \cdot\left(\frac{\partial z_{k}(t)^{T}}{\partial x(t)} A_{z} x\right)\left[\begin{array}{cc}
0 & \left(G_{z}^{k}\right)^{T} \\
G_{z}^{k} & 0
\end{array}\right]>0
\end{aligned}
$$

Inequality (14) is therefore implied by

$\left[\begin{array}{cc}P_{z} & F_{z}^{T} \\ F_{z} & \mu^{2} I_{m}\end{array}\right]+\sum_{k=1}^{p} \sum_{v=1}^{n} \sum_{s=1}^{n}\left(A_{z}\right)_{v s} \frac{\partial w_{0}^{k}}{\partial x_{v}} x_{s}\left[\begin{array}{cc}0 & \left(G_{z}^{k}\right)^{T} \\ G_{z}^{k} & 0\end{array}\right]>0$

which as expression (13) depends on bounding terms $\left|\frac{\partial w_{0}^{k}}{\partial x_{v}} x_{s}\right|$ to yield LMI expressions.

Two choices are offered to deal with these bounds, based respectively on the following properties:

$$
X+\left(\frac{\partial w_{0}^{k}}{\partial x_{v}} x_{s}\right) Y+\left(\frac{\partial w_{0}^{k}}{\partial x_{v}} u_{e}\right) Z \leq 0 \Leftarrow X \pm \lambda_{k s v} \times Y \pm \eta_{k v} \times \mu Z \leq 0
$$

or 
$Q=Q^{T}>0, S=S^{T}>0$

$X+\left(\frac{\partial w_{0}^{k}}{\partial x_{v}} x_{s}\right) Y+\left(\frac{\partial w_{0}^{k}}{\partial x_{v}} u_{e}\right) Z \leq X+\frac{1}{2}\left(\lambda_{k s v}^{2} Q+Y Q^{-1} Y\right)+\frac{1}{2}\left(\eta_{k v}^{2} S+\mu^{2} Z S^{-1} Z\right)<0$

By means of property (16) the following result is obtained:

Theorem 2: If there exist matrices of proper size $P_{j}=P_{j}^{T} \geq c^{2} I, F_{j}, G_{j}^{k}, \forall j \in\{1, \ldots, r\}, k \in\{1, \ldots, p\}$ such that LMIs

$\tilde{\Lambda}_{\mathbf{a}}^{\alpha}=\sum_{\substack{\mathbf{b} \in \mathbf{A}(\mathbf{a}) \\ i j l=b_{1} b_{2} b_{3}}} \Lambda_{i j l}^{\alpha}<0, \quad \forall \mathbf{a} \in \mathbf{I}_{q}^{+}, \alpha \in\left\{1, \cdots, 2^{p n(m+n)}\right\}$

hold for a given $q \geq 3$ with

$\Lambda_{i j l}^{\alpha}=\operatorname{block}-\operatorname{diag}\left[\Upsilon_{i j l}^{\alpha}, \Omega_{i j}^{\alpha}\right]$,

$\Upsilon_{i j l}^{\alpha}=A_{i} P_{j}+B_{i} F_{j}+P_{j} A_{i}^{T}+F_{j}^{T} B_{i}^{T}$

$-\sum_{k=1}^{p} \sum_{v=1}^{n} \sum_{e=1}^{m}(-1)^{d_{k v(e+n)}^{\alpha}} \eta_{k v} \mu\left(B_{i}\right)_{v e}\left(P_{g_{1}(j, k)}-P_{g_{2}(j, k)}\right)$

$-\sum_{k=1}^{p} \sum_{v=1}^{n} \sum_{s=1}^{n}(-1)^{d_{k v s}^{\alpha}} \lambda_{k v s}\left(A_{i}\right)_{v s}\left(P_{g_{1}(j, k)}-P_{g_{2}(j, k)}-B_{l} G_{j}^{k}-\left(G_{j}^{k}\right)^{T} B_{l}^{T}\right)$

$\Omega_{i j}^{\alpha}=-\left[\begin{array}{cc}P_{j} & F_{j}^{T} \\ F_{j} & \mu^{2} I_{m}\end{array}\right]-\sum_{k=1}^{p} \sum_{v=1}^{n} \sum_{s=1}^{n}(-1)^{d_{k v s}^{\alpha}} \lambda_{k v s}\left(A_{i}\right)_{v s}\left[\begin{array}{cc}0 & \left(G_{j}^{k}\right)^{T} \\ G_{j}^{k} & 0\end{array}\right]$,

$d_{k v(e+n)}^{\alpha}, \quad d_{k v s}^{\alpha}$ defined from $\alpha-1=d_{p n(n+m)}^{\alpha}+d_{p n(n+m-1)}^{\alpha} \times 2+\cdots+d_{111}^{\alpha} \times 2^{p n(n+m)-1}$, and $g_{1}(j, k), \quad g_{2}(j, k)$

defined as in (12), then $x(t),|x(0)|<c$ tends to zero exponentially for any trajectory satisfying (6) in the

outermost Lyapunov level contained in $R_{o} \subseteq(R \cap C)$ with $R=\bigcap_{e, k, s, v}\left\{x:\left|\frac{\partial w_{0}^{k}}{\partial x_{v}} u_{e}\right| \leq \mu \eta_{k v},\left|\frac{\partial w_{0}^{k}}{\partial x_{v}} x_{s}\right| \leq \lambda_{k v s}\right\}$. 
Proof: From relaxation Lemma in (8), LMIs in (18) imply $\Lambda_{z z z}^{\alpha}=$ block $-\operatorname{diag}\left[\Upsilon_{z z z}^{\alpha}, \Omega_{z z z}^{\alpha}\right]<0$, or equivalently,

$$
\begin{aligned}
\Upsilon_{z z z}^{\alpha}= & A_{z} P_{z}+B_{z} F_{z}+P_{z} A_{z}^{T}+F_{z}^{T} B_{z}^{T}-\sum_{k=1}^{p} \sum_{v=1}^{n} \sum_{e=1}^{m}(-1)^{d_{k v(c+n)}^{\alpha}} \eta_{k v} \mu\left(B_{z}\right)_{v e}\left(P_{g_{1}(z, k)}-P_{g_{2}(z, k)}\right) \\
& -\sum_{k=1}^{p} \sum_{v=1}^{n} \sum_{s=1}^{n}(-1)^{d_{k v s}^{\alpha}} \lambda_{k v s}\left(A_{z}\right)_{v s}\left(P_{g_{1}(z, k)}-P_{g_{2}(z, k)}-B_{z} G_{z}^{k}-\left(G_{z}^{k}\right)^{T} B_{z}^{T}\right)<0, \\
\Omega_{z z}^{\alpha}= & -\left[\begin{array}{cc}
P_{z} & F_{z}^{T} \\
F_{z} & \mu^{2} I_{m}
\end{array}\right]-\sum_{k=1}^{p} \sum_{v=1}^{n} \sum_{s=1}^{n}(-1)^{d_{k v s}^{\alpha}} \lambda_{k v s}\left(A_{z}\right)_{v s}\left[\begin{array}{cc}
0 & \left(G_{z}^{k}\right)^{T} \\
G_{z}^{k} & 0
\end{array}\right]<0 .
\end{aligned}
$$

Given that all the possible sign combinations of the terms involving $\eta_{k v}$ and $\lambda_{k v s}$ are taken into account in the previous expressions by means of parameter $\alpha$, and provided that $\left|\frac{\partial w_{0}^{k}}{\partial x_{v}} u_{e}\right| \leq \mu \eta_{k v},\left|\frac{\partial w_{0}^{k}}{\partial x_{v}} x_{s}\right| \leq \lambda_{k v s}$ in $R_{o}$, it follows that:

$$
\begin{aligned}
& A_{z} P_{z}+B_{z} F_{z}+P_{z} A_{z}^{T}+F_{z}^{T} B_{z}^{T}-\sum_{k=1}^{p} \sum_{v=1}^{n} \sum_{e=1}^{m} \frac{\partial w_{0}^{k}}{\partial x_{v}} u_{e}\left(B_{z}\right)_{v e}\left(P_{g_{1}(z, k)}-P_{g_{2}(z, k)}\right) \\
& \quad-\sum_{k=1}^{p} \sum_{v=1}^{n} \sum_{s=1}^{n}\left(A_{z}\right)_{v s} \frac{\partial w_{0}^{k}}{\partial x_{v}} x_{s}\left(P_{g_{1}(z, k)}-P_{g_{2}(z, k)}-B_{z} G_{z}^{k}-\left(G_{z}^{k}\right)^{T} B_{z}^{T}\right)<\Upsilon_{z z z}^{\alpha}<0 \\
& -\left[\begin{array}{cc}
P_{z} & F_{z}^{T} \\
F_{z} & \mu^{2} I_{m}
\end{array}\right]-\sum_{k=1}^{p} \sum_{v=1}^{n} \sum_{s=1}^{n}\left(A_{z}\right)_{v s} \frac{\partial w_{0}^{k}}{\partial x_{v}} x_{s}\left[\begin{array}{cc}
0 & \left(G_{z}^{k}\right)^{T} \\
G_{z}^{k} & 0
\end{array}\right]<\Omega_{z z}^{\alpha}<0 .
\end{aligned}
$$

The time-derivative (10) of the non-quadratic Lyapunov function candidate (9) is negative if (11) holds, which is implied by condition (19). Moreover, $\|u\|_{2}<\mu$ holds if (15) does so, which is guaranteed by condition (20), thus concluding the proof.

A similar result is now presented via property (17): 
Theorem 3: If there exist matrices of proper size $P_{j}=P_{j}^{T} \geq c^{2} I, F_{j}, G_{j}^{k}, Q_{i j l}^{k v s}>0, S_{i j l}^{k v e}>0$, and $T_{i j}^{k v s}>0$, $\forall i, j, l \in\{1, \ldots, r\}, k \in\{1, \ldots, p\}, s, v \in\{1, \ldots, n\}, e \in\{1, \ldots, m\}$, such that LMIs

$$
\tilde{\Lambda}_{\mathbf{a}}=\sum_{\substack{\mathbf{b} \in \mathcal{A}_{(\mathbf{a}} b_{3} \\ i j l=b_{1} b_{2} b_{3}}} \Lambda_{i j l}<0, \quad \forall \mathbf{a} \in \mathbf{I}_{q}^{+},
$$

hold for a given $q \geq 3$ with $\Lambda_{i j l}=$ block $-\operatorname{diag}\left[\Upsilon_{i j l}, \Omega_{i j}\right]$,

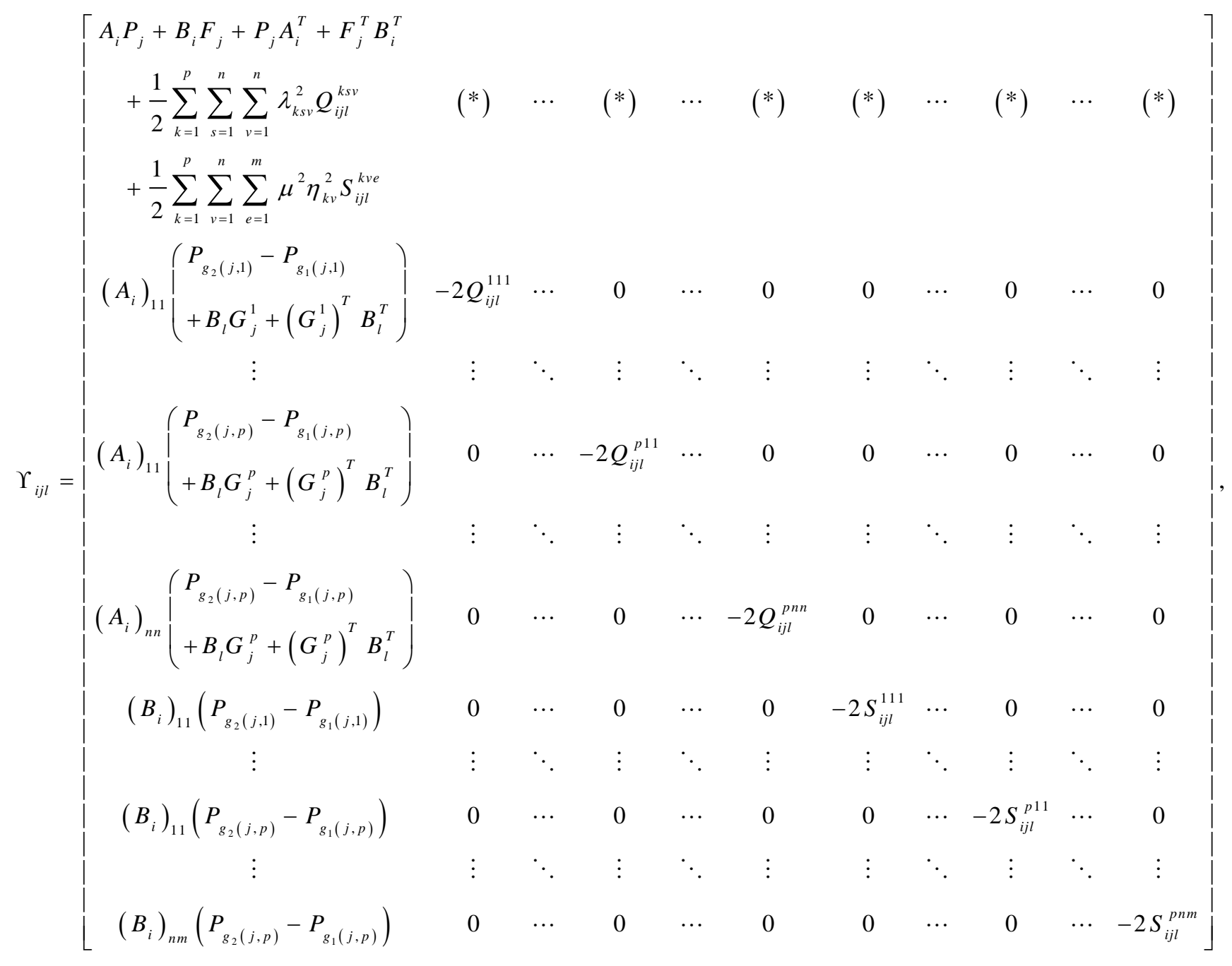




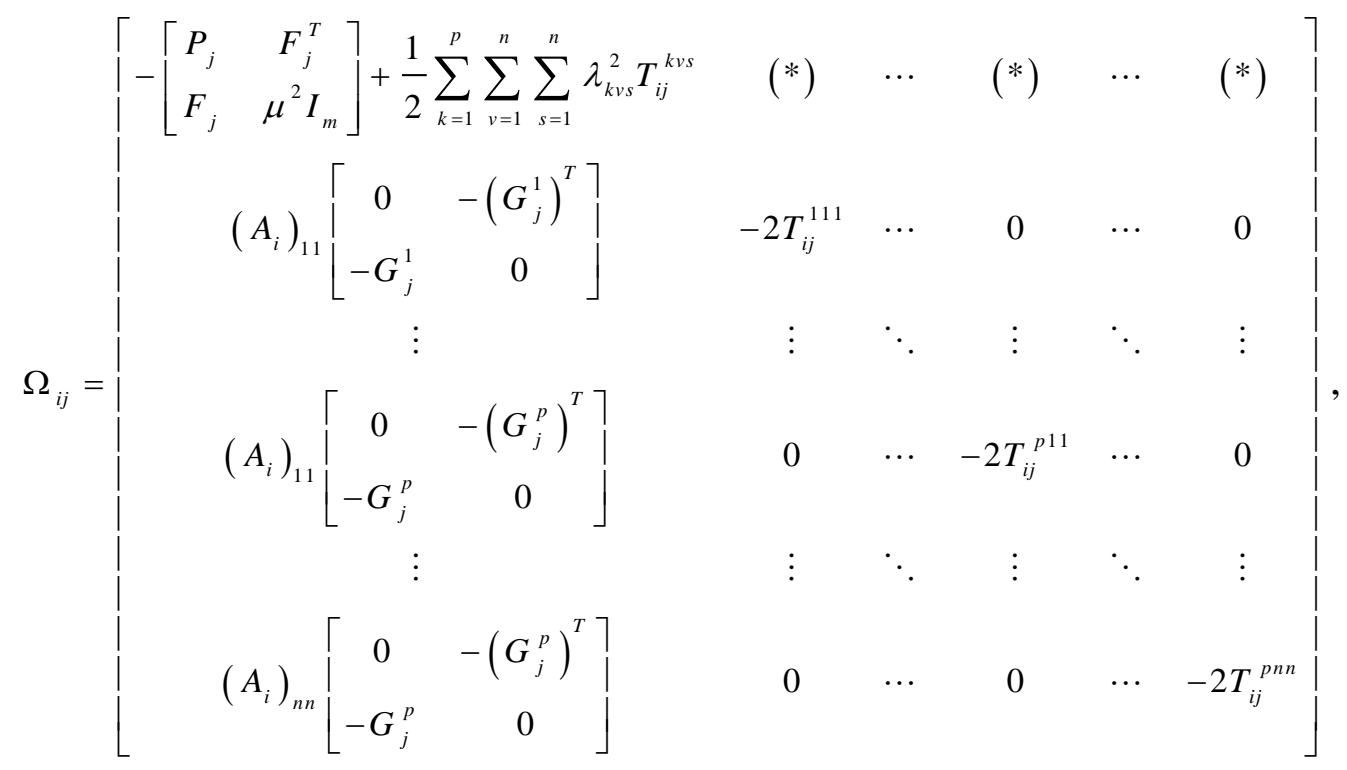

$g_{1}(\alpha, k), g_{2}(\alpha, k)$ defined as in (12), then $x(t)$ tends to zero exponentially for any trajectory satisfying

(6) in the outermost Lyapunov level $R_{o} \subseteq(R \cap C)$ with $R=\bigcap_{e, k, s, v}\left\{x:\left|\frac{\partial w_{0}^{k}}{\partial x_{v}} u_{e}\right| \leq \mu \eta_{k v},\left|\frac{\partial w_{0}^{k}}{\partial x_{v}} x_{s}\right| \leq \lambda_{k v s}\right\}$.

Proof: From (17) it is clear that

$$
\begin{aligned}
& A_{z} P_{z}+B_{z} F_{z}+P_{z} A_{z}^{T}+F_{z}^{T} B_{z}^{T}-\sum_{k=1}^{p} \sum_{v=1}^{n} \sum_{e=1}^{m} \frac{\partial w_{0}^{k}}{\partial x_{v}} u_{e}\left(B_{z}\right)_{v e}\left(P_{g_{1}(z, k)}-P_{g_{2}(z, k)}\right) \\
& \quad-\sum_{k=1}^{p} \sum_{v=1}^{n} \sum_{s=1}^{n}\left(A_{z}\right)_{v s} \frac{\partial w_{0}^{k}}{\partial x_{v}} x_{s}\left(P_{g_{1}(z, k)}-P_{g_{2}(z, k)}-B_{z} G_{z}^{k}-\left(G_{z}^{k}\right)^{T} B_{z}^{T}\right) \\
& =A_{z} P_{z}+B_{z} F_{z}+P_{z} A_{z}^{T}+F_{z}^{T} B_{z}^{T} \\
& \quad+\sum_{k=1}^{p} \sum_{v=1}^{n} \sum_{e=1}^{m} \frac{\partial w_{0}^{k}}{\partial x_{v}} u_{e}\left(B_{z}\right)_{v e}\left(P_{g_{2}(z, k)}-P_{g_{1}(z, k)}\right) \\
& \quad+\sum_{k=1}^{p} \sum_{v=1}^{n} \sum_{s=1}^{n}\left(A_{z}\right)_{v s} \frac{\partial w_{0}^{k}}{\partial x_{v}} x_{s}\left(P_{g_{2}(z, k)}-P_{g_{1}(z, k)}+B_{z} G_{z}^{k}+\left(G_{z}^{k}\right)^{T} B_{z}^{T}\right)
\end{aligned}
$$




$$
\begin{aligned}
\leq & A_{z} P_{z}+B_{z} F_{z}+P_{z} A_{z}^{T}+F_{z}^{T} B_{z}^{T} \\
& +\frac{1}{2} \sum_{k=1}^{p} \sum_{v=1}^{n} \sum_{e=1}^{m}\left[\mu^{2} \eta_{k v}^{2} S_{z z z}^{k v e}+\left(B_{z}\right)_{v e}^{2}\left(P_{g_{2}(z, k)}-P_{g_{1}(z, k)}\right)\left(S_{z z z}^{k v e}\right)^{-1}\left(P_{g_{2}(z, k)}-P_{g_{1}(z, k)}\right)\right] \\
& +\frac{1}{2} \sum_{k=1}^{p} \sum_{v=1}^{n} \sum_{s=1}^{n}\left[\lambda_{k v s}^{2} Q_{z z z}^{k v s}+\left(A_{z}\right)_{v s}^{2}\left(P_{g_{2}(z, k)}-P_{g_{1}(z, k)}+B_{z} G_{z}^{k}+\left(G_{z}^{k}\right)^{T} B_{z}^{T}\right)\left(Q_{z z z}^{k v s}\right)^{-1}\right. \\
& \left.\left(P_{g_{2}(z, k)}-P_{g_{1}(z, k)}+B_{z} G_{z}^{k}+\left(G_{z}^{k}\right)^{T} B_{z}^{T}\right)\right]<0
\end{aligned}
$$

Successively applying the Schur complement to the previous expression leads to

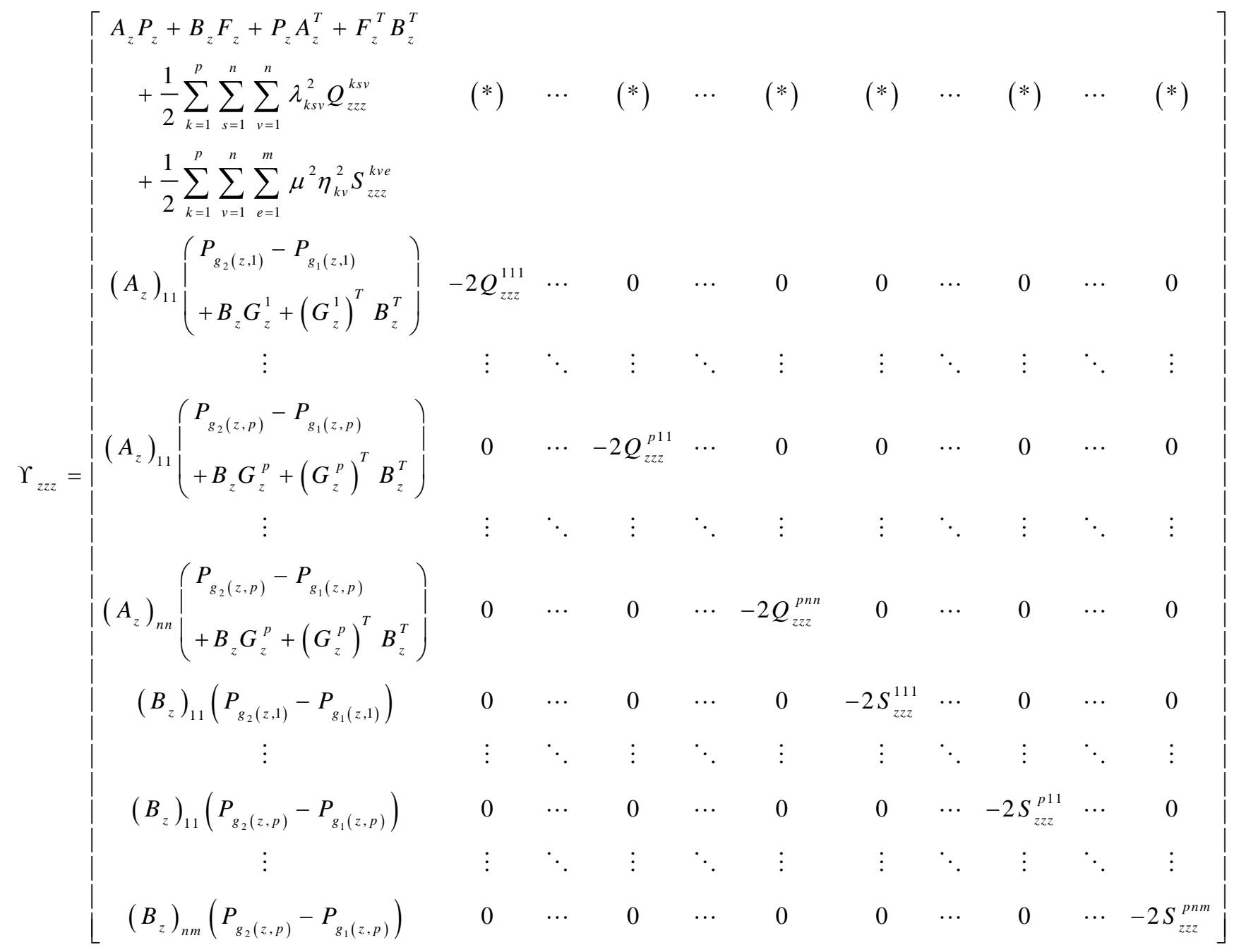

A similar procedure shows that applying Schur complement to the last inequality in 


$$
\begin{aligned}
& -\left[\begin{array}{cc}
P_{z} & F_{z}^{T} \\
F_{z} & \mu^{2} I_{m}
\end{array}\right]-\sum_{k=1}^{p} \sum_{v=1}^{n} \sum_{s=1}^{n}\left(A_{z}\right)_{v s} \frac{\partial w_{0}^{k}}{\partial x_{v}} x_{s}\left[\begin{array}{cc}
0 & \left(G_{z}^{k}\right)^{T} \\
G_{z}^{k} & 0
\end{array}\right] \\
& \quad=-\left[\begin{array}{cc}
P_{z} & F_{z}^{T} \\
F_{z} & \mu^{2} I_{m}
\end{array}\right]+\sum_{k=1}^{p} \sum_{v=1}^{n} \sum_{s=1}^{n}\left(A_{z}\right)_{v s} \frac{\partial w_{0}^{k}}{\partial x_{v}} x_{s}\left[\begin{array}{cc}
0 & \left.-\left(G_{z}^{k}\right)^{T}\right] \\
-G_{z}^{k} & 0
\end{array}\right] \\
& \leq-\left[\begin{array}{cc}
P_{z} & F_{z}^{T} \\
F_{z} & \mu^{2} I_{m}
\end{array}\right]+\frac{1}{2} \sum_{k=1}^{p} \sum_{v=1}^{n} \sum_{s=1}^{n}\left[\lambda_{k v s}^{2} T_{z z}^{k v s}+\left(A_{z}\right)_{v s}^{2}\left[\begin{array}{cc}
0 & -\left(G_{z}^{k}\right)^{T} \\
-G_{z}^{k} & 0
\end{array}\right]\left[T_{z z}^{k v s}\right]^{-1}\left[\begin{array}{cc}
0 & \left.\left.-\left(G_{z}^{k}\right)^{T}\right]\right]<0 \\
-G_{z}^{k} & 0
\end{array}\right]\right]<
\end{aligned}
$$

gives

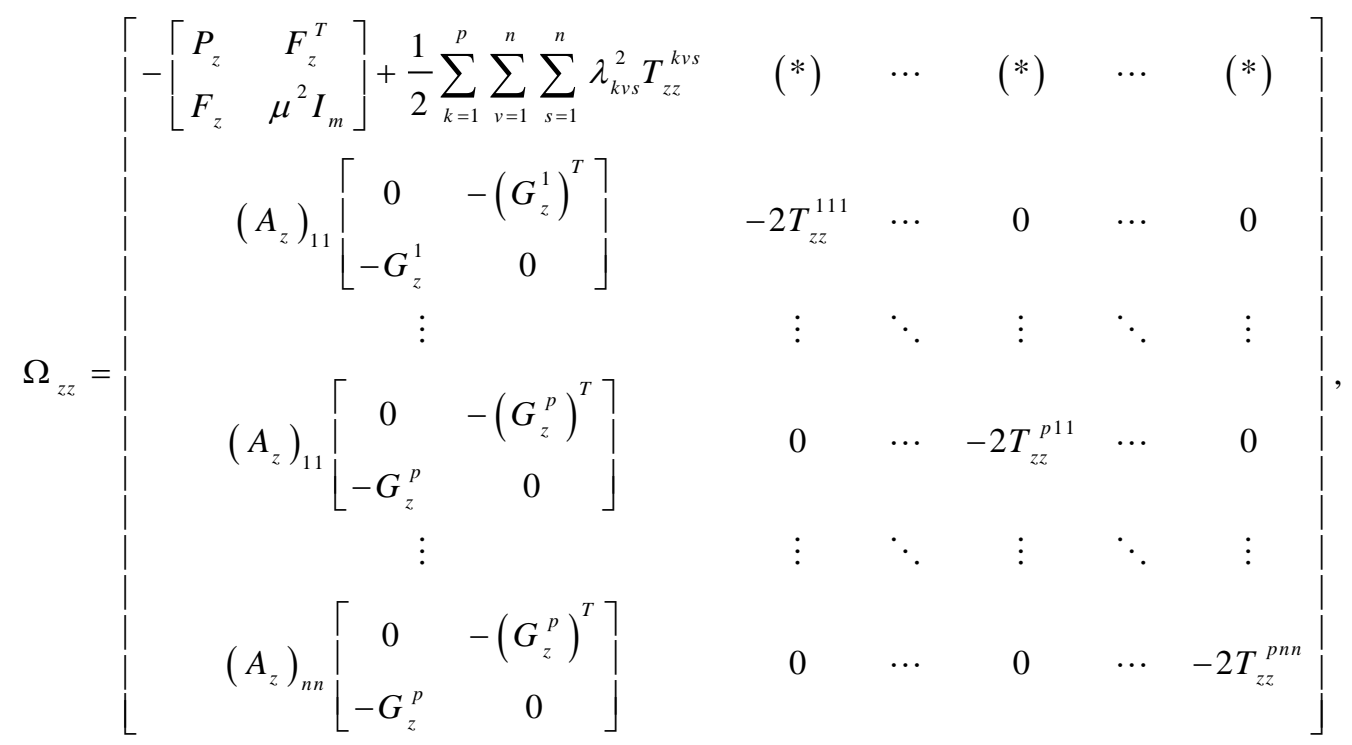

which allows defining $\Lambda_{z z z}=$ block $-\operatorname{diag}\left[\Upsilon_{z z z}, \Omega_{z z}\right]$.

Applying Relaxation Lemma (8) to $\Lambda_{z z z}$ leads to LMIs (21), thus concluding the proof.

Remark 1: Theorems 2 and 3 provide LMI conditions for local non-quadratic controller design of continuous-time TS models. The control law thus designed is guaranteed to stabilize the TS model in the outermost Lyapunov level of region $R$, whose bounds depend on those of the compact set $C$, the designed bound $\mu$ of the input, and the inherited bounds of the partial derivatives $\partial w_{0}^{k} / \partial x_{v}$. Let us recall that, in many previous studies, see e.g. [13,19,21-25,27], controller design conditions have been obtained by 
making very restrictive assumptions of the sort $\left\|\dot{P}_{z}\right\|<\phi_{z}$. It means that these bounds cannot be known in advance since they depend on the time-derivative of the state, which cannot be assured to be a priori stable. Therefore, all these previous methods fail to give a solution to this problem whereas the parameters $\left|\frac{\partial w_{0}^{k}}{\partial x_{v}} x_{s}\right|$ and $\left|\frac{\partial w_{0}^{k}}{\partial x_{v}} u_{e}\right|$, which are used in the above proposed theorems, are always known. Indeed, it is wellknown that the quasi-LPV (or TS) description is only validated in a compact set of the state space, therefore $x_{s}$ is known and the functions $w_{0}^{k}$ being smooth, their derivative $\partial w_{0}^{k} / \partial x_{v}$ limits are easy to compute. Moreover, considering any practical application, conditions (15) allows to guarantee $\left\|_{u}\right\|_{2}<\mu$ so as to bound $\left|\frac{\partial w_{0}^{k}}{\partial x_{v}} u_{e}\right|$. The parameter $\mu$ (which can be obtained from the actuators technical characteristics) ensure that, with the obtained solution to LMIs, the control signal will always be bounded while the initial conditions belong inside the domain of attraction $D$. Therefore, the proposed study provides an answer to previous non-quadratic approaches where unknown parameters had to be assumed for the LMI computation. At last, it is important to point out that most of the previous studies dealing with TS systems tend to explore global stability regardless of their local nature. By contrast, local analysis and controller design for nonlinear systems are widely used; in this sense the proposed approach better fits the nonlinear spirit by reducing global objectives to local stability conditions for TS models.

Remark 2: Conditions in Theorems 2 and 3 reduce to those of the quadratic global controller design if $P_{i}=P, G_{i}^{k}=0, i \in\{1, \ldots, r\}, k \in\{1, \ldots, p\}$. For Theorem 2 it cancels the last two triple sums of $\Upsilon_{i j l}^{\alpha}$ and the last triple sum of $\Omega_{i j}^{\alpha}$ in (18), allowing $\lambda_{k v s}$ and $\eta_{k v}$ to grow arbitrarily large (global controller). As for Theorem 3, it makes zero all the non-diagonal terms of $\Upsilon_{i j l}$ and $\Omega_{i j}$ in (21), so matrices $Q_{i j l}^{k s v}>0, S_{i j l}^{k v e}>0$, and $T_{i j}^{k s v}>0$ can always be found to fulfill conditions (21) despite the values of $\lambda_{k v s}$ and $\eta_{k v}$.

Remark 3: Even if conditions in Theorems 2 or 3 fail to be fulfilled for a given set of constants $\lambda_{k s v}, \eta_{k v}>0$ 
(i.e., for a given region), the largest region of attraction can still be found via linear programming by testing conditions in these theorems for successively larger values of a common factor $\lambda>0$ multiplying both sets of constants, assuming that conditions in Theorem 1 hold.

Remark 4: Notice that compared with the quadratic case, theorem 2 increases the number of LMI constraints from $r+1$ to $r+r^{2} \times 2^{p n-1}$ whereas theorem 3 increases them from $r+1$ to $r+r^{2}$ while increasing the size of each LMI from $\square^{n \times n}$ to $\square^{(n \times k \times u) \times(n \times k \times u)}$.

\section{Examples}

Example 1: Consider the following TS model [28]

$\dot{x}=\sum_{i=1}^{2} h_{i}\left(x_{1}\right)\left(A_{i} x+B_{i} u\right)$

with $\quad h_{1}\left(x_{1}\right)=\frac{1-\sin \left(x_{1}\right)}{2}, \quad h_{2}\left(x_{1}\right)=\frac{1+\sin \left(x_{1}\right)}{2}, \quad A_{1}=\left[\begin{array}{cc}2 & -10 \\ 2 & 0\end{array}\right], \quad A_{2}=\left[\begin{array}{cc}a & -5 \\ 1 & 2\end{array}\right], \quad B_{1}=\left[\begin{array}{ll}1 \\ 1\end{array}\right], \quad B_{2}=\left[\begin{array}{l}b \\ 2\end{array}\right]$,

$\left|x_{i}\right|<\frac{\pi}{2},-20 \leq a \leq 10,0 \leq b \leq 25$.

Figure 1 shows the effects of parameter $\mu$ in a single stabilization case $(a=4.6, b=0)$. Conditions in Theorem 2 and linear programming have been combined to find local controllers maximizing the region of attraction for $\mu=30$ (bounded by the dashed closed curve) and $\mu=100$ (bounded by the solid closed curve). As expected, the latter provides the biggest region and includes the former one. The bounds of $R=\bigcap_{e, k, s, v}\left\{x:\left|\frac{\partial w_{0}^{k}}{\partial x_{v}} u_{e}\right| \leq \mu \eta_{k v},\left|\frac{\partial w_{0}^{k}}{\partial x_{v}} x_{s}\right| \leq \lambda_{k v s}\right\}$ for $\mu=100$ are shown in dotted lines.

Figure 2 shows the advantages of using gains $G_{i}{ }^{k}$ for the previous case $(a=4.6, b=0)$. Theorem 2 and linear programming have been used again to find local controllers maximizing the region of attraction 
without $G_{i}^{k}$ (bounded by a dashed closed curve) and including $G_{i}^{k}$ (bounded by the solid closed curve). It is clear that the new control law (5) outperforms the existing non-quadratic ones [22]. As in Figure 1, the bounds of region $\mathrm{R}$ for the case including $G_{i}^{k}$ are shown in dotted lines.

Conditions in [21] are unfeasible for this model in any point of the considered grid. The proposed approach is therefore compared with that in [28], for which the following pole placement LMIs for a D-region must be included:

$\left[\begin{array}{cc}-\bar{r} P_{i} & \bar{G}_{i i j}^{\alpha} \\ (*) & -\bar{r}_{i}\end{array}\right]<0,\left[\begin{array}{ll}\sin \theta\left(\bar{G}_{i i j}^{\alpha}+\left(\bar{G}_{i i j}^{\alpha}\right)^{T}\right) & \cos \theta\left(\bar{G}_{i i j}^{\alpha}-\left(\bar{G}_{i i j}^{\alpha}\right)^{T}\right) \\ \cos \theta\left(\bar{G}_{i i j}^{\alpha}-\left(\bar{G}_{i i j}^{\alpha}\right)^{T}\right) & \sin \theta\left(\bar{G}_{i i j}^{\alpha}+\left(\bar{G}_{i i j}^{\alpha}\right)^{T}\right)\end{array}\right]<0, \bar{G}_{i i j}^{\alpha}+\left(\bar{G}_{i i j}^{\alpha}\right)^{T}+\beta P_{i}<0$,

with $\bar{G}_{i i j}^{\alpha}=A_{i} P_{i}+B_{i}\left(F_{i}+\sum_{k=1}^{p} \sum_{v=1}^{n} \sum_{s=1}^{n}\left(A_{j}\right)_{v s}(-1)^{d_{k v}^{\alpha}} \lambda_{k s v} G_{i}^{k}\right)<0, i, j \in\{1, \cdots, r\}, \alpha \in\left\{1, \cdots, 2^{p n^{2}}\right\}$, and $\bar{r}, \theta$ and $\beta$ as parameters defining the $\mathrm{D}$-region.

The proposed approach is $\mu$-dependent $(|u|<\mu)$. Figure 3 shows the effect of this parameter on the stabilizability region size for $-20 \leq a \leq 10,0 \leq b \leq 25$. As $\mu$ increases, more feasible points ("+" marks) are found via conditions in Theorem 1 and (23); these points are then compared with those of the quadratic approach ("o" marks) and those of the line integral approach in [28] ("x" marks). Note that with $\mu=500$, the new approach includes any feasible point of the other approaches considered.

Example 2: Consider the following TS model [21]:

$\dot{x}=\sum_{i=1}^{2} h_{i}\left(x_{1}\right)\left(A_{i} x+B_{i} u\right)$,

with $h_{1}\left(x_{1}\right)=\frac{1+\sin \left(x_{1}\right)}{2}, \quad h_{2}\left(x_{1}\right)=\frac{1-\sin \left(x_{1}\right)}{2}, \quad A_{1}=\left[\begin{array}{cc}-5 & -4 \\ -1 & -2\end{array}\right], \quad A_{2}=\left[\begin{array}{cc}-2 & -4 \\ 20 & -2\end{array}\right], \quad B_{1}=\left[\begin{array}{c}0 \\ 10\end{array}\right], \quad B_{2}=\left[\begin{array}{l}0 \\ 3\end{array}\right]$, 
$\left|x_{i}\right|<\frac{\pi}{2}$

The approach in [21] has several drawbacks, none of which is shared by the proposed one: it only applies for TS models whose membership functions have strictly state-dependent time-derivatives (like (24)); its conditions are not LMIs unless some parameters are given; its proposed control law includes timederivatives of the membership functions which are not always available. For the sake of comparison between Theorems 2 and 3, they have been applied for stabilization of TS model (24) for different values of $\mu$ and $c$, with $|u|<\mu,|x(0)|<c$; the resulting maxima $\lambda_{k v s}$ and $\eta_{k v}$ are summarized in Table 1. Figure 4 illustrates the states evolution of TS model (24) under a control law (5) whose gains have been obtained via Theorem 3 with $\mu=0.1, \quad c=\sqrt{2} \pi / 2$; correspondingly, Figure 5 shows the control law evolution corresponding to this case.

\section{Conclusions and Perspectives}

A novel approach for local non-quadratic stabilization of continuous-time TS models has been presented. Thanks to the information provided by the MFs and a proper manipulation of their time-derivatives, a new non-PDC control law has been proposed which locally stabilizes a continuous-time TS model through straightforward LMI conditions. It has been shown that reducing global goals to the estimation of a region of attraction constitutes a good way-out from the quadratic framework, since it provides new basis to overcome old issues on stabilization of continuous-time TS models while incorporating former results as particular cases. Two examples have been provided that clearly show that the proposed approach outperforms the palliative solutions of the previous non-quadratic studies.

\section{Acknowledgment}

The present research work has been supported by International Campus on Safety and Intermodality in 
Transportation, the Nord-Pas-de-Calais Region, the European Community, the Regional Delegation for

Research and Technology, the Ministry of Higher Education and Research, and the National Center for Scientific Research. The authors gratefully acknowledge the support of these institutions.

\section{References}

[1] T. Takagi and M. Sugeno, Fuzzy identification of systems and its application to modeling and control, IEEE Trans. on System Man and Cybernetics. 15(1) (1985) 116-132.

[2] T. Taniguchi, K. Tanaka, H.O. Wang, Model construction, rule reduction and robust compensation for generalized form of Takagi-Sugeno fuzzy systems, IEEE Trans. on Fuzzy Systems. 9(4) (2001) 525537.

[3] H.O. Wang, K. Tanaka, M. Griffin, An approach to fuzzy control of nonlinear systems: Stability and Design Issues, IEEE Trans. on Fuzzy Systems. 4(1) (1996) 14-23.

[4] S. Boyd, L. El Ghaoui, E. Féron, V. Balakrishnan, Linear matrix inequalities in system and control theory. Studies in Applied Mathematics; Philadelphia, USA, 1994.

[5] K. Tanaka and H.O. Wang, Fuzzy control systems design and analysis. A linear matrix inequality approach. John Wiley and Sons, New York. 2001.

[6] T. Senthilkumar, P. Balasubramaniam, Delay-dependent robust Ho control for uncertain stochastic TS fuzzy systems with time-varying state and input delays, International Journal of Systems Science. 42 (5) (2011) 877-887.

[7] T. Senthilkumar, P. Balasubramaniam, Robust $\mathrm{H} \infty$ control for nonlinear uncertain stochastic T-S fuzzy systems with time-delays, Applied Mathematics Letters. 24(12) (2011) 1986-1994.

[8] M. Zerar, K. Guelton, N. Manamanni, Linear Fractional Transformation Based H-infinity Output Stabilization for Takagi-Sugeno Fuzzy Models, Mediterranean Journal of Measurement and Control. 4(3) (2008) 111-121. 
[9] T. Bouarar, K. Guelton, N. Manamanni, LMI stability conditions for Takagi-Sugeno uncertain descriptors, In Proc. in Proc. of the IEEE International Fuzzy Systems Conference, FUZZ-IEEE 2007, London, UK, 2007.

[10]A. Sala, T.M. Guerra, R. Babuska, Perspectives of fuzzy systems and control, Fuzzy Sets \& Systems. 156 (2005) 432-444.

[11]T.M. Guerra, M. Bernal, A. Kruszewski, M. Afroun, A way to improve results for the stabilization of continuous-time fuzzy descriptor models. In Proc. of 46th IEEE CDC, 5960-5964, New Orleans, USA, 2007.

[12]T. Bouarar, K. Guelton, N. Manamanni, Robust fuzzy Lyapunov stabilization for uncertain and disturbed Takagi-Sugeno descriptors, ISA Transactions. 49(4) (2010) 447-461.

[13]H.D. Tuan, P. Apkarian, T. Narikiyo, Y. Yamamoto, Parameterized linear matrix inequality techniques in fuzzy control system design, IEEE Trans. on Fuzzy Systems. 9(2) (2001) 324-332.

[14] A. Sala, C. Ariño, Asymptotically necessary and sufficient conditions for stability and performance in fuzzy control: Applications of Polya's theorem; Fuzzy Sets and Systems. 158(24) (2007) 2671-2686.

[15] A. Sala, C. Ariño, Relaxed stability and performance conditions for Takagi-Sugeno fuzzy systems with knowledge on membership-function overlap, IEEE Trans. SMC(B). 37(3) (2007) 727-732.

[16] A. Sala, T.M. Guerra, Stability Analysis of Fuzzy Systems: membership-shape and polynomial approaches, In Proc. of IFAC World Congress, Seoul, South Korea, 5605-5610, 2008.

[17] M. Bernal, T.M. Guerra, A. Kruszewski, A membership-function-dependent approach for stability analysis and controller synthesis of Takagi-Sugeno models, Fuzzy Sets \& Systems. 160(19) (2009) 2776-2795.

[18] M. Johansson, A. Rantzer, K. Arzen, Piecewise quadratic stability of fuzzy systems, IEEE Trans. on Fuzzy Systems. 7 (1999) 713-722. 
[19] K. Tanaka, T. Hori, H.O. Wang, A fuzzy Lyapunov approach to fuzzy control systems design, in Proc. American Control Conference, Arlington, USA, 4790-4795, 2001.

[20] G. Feng, C.L. Chen, D. Soun, Y. Zhu, Hळ controller synthesis of fuzzy dynamic systems based on piecewise Lyapunov functions and bilinear matrix inequalities. IEEE Trans. on Fuzzy Systems. 13(1) (2005) 94-103.

[21] K. Tanaka, T. Hori, H.O. Wang, A multiple Lyapunov function approach to stabilization of fuzzy control systems, IEEE Trans. on Fuzzy Systems. 11(4) (2003) 582-589.

[22] T.M. Guerra, L. Vermeiren, LMI-based relaxed non-quadratic stabilization conditions for nonlinear systems in Takagi-Sugeno's form, Automatica. 40(5) (2004) 823-829.

[23] W.J. Wang, C.H. Sun, Relaxed stability and stabilization conditions for a T-S fuzzy discrete system, Fuzzy Sets \& Systems. 156(2) (2005) 208-225.

[24] B.C. Ding, H.X. Sun, P. Yang, Further studies on LMI-based relaxed stabilization conditions for nonlinear systems in Takagi-Sugeno’s form, Automatica. 42 (2006) 503-508.

[25] A. Kruszewski, R. Wang, T.M. Guerra, Non-quadratic stabilization conditions for a class of uncertain non linear discrete-time T-S fuzzy models: a new approach, IEEE Trans. on Automatic Control. 53(2) (2008) 606-611.

[26] T.M. Guerra, A. Kruszewski, M. Bernal, Control Law Proposition for the Stabilization of Discrete Takagi-Sugeno Models, IEEE Trans. on Fuzzy Systems. 17(3) (2009) 724-731.

[27] Y. Blanco, W. Perruquetti, P.Borne, Stability and stabilization of nonlinear systems and TanakaSugeno fuzzy models, in Proc. of the European Control Conference, Lisbon, Portugal, 2001.

[28] B.J. Rhee, S. Won, A new fuzzy Lyapunov function approach for a Takagi-Sugeno fuzzy control system design, Fuzzy Sets \& Systems. 157(9) (2006) 1211-1228. 
[29] K. Guelton, T.M. Guerra, M. Bernal, T. Bouarar, N. Manamanni, Comments on Fuzzy Control Systems Design via Fuzzy Lyapunov Functions, IEEE Trans. on Systems, Man, and Cybernetics (B). 40(3) (2010) 970-973.

[30] T.M. Guerra, M. Bernal, A way to escape from the quadratic framework, in Proc. FUZZ-IEEE Conference, Jeju, Korea, 2009.

[31] H. Khalil, Nonlinear Systems, $3^{\text {rd }}$ edition. Prentice Hall, New Jersey, USA. 2002.

[32] G. Chesi, A. Garulli, A. Tesi, A. Vicino, Homogeneous Polynomial Forms for Robustness Analysis of Uncertain Systems, Lecture Notes in Control and Information Sciences, volume 390, Springer, 2009.

[33] M. Bernal, T.M. Guerra, Generalized Nonquadratic Stability of Continuous-Time Takagi-Sugeno Models, IEEE Trans. on Fuzzy Systems. 18(4) (2010) 815-822. 


\section{Figures and table}

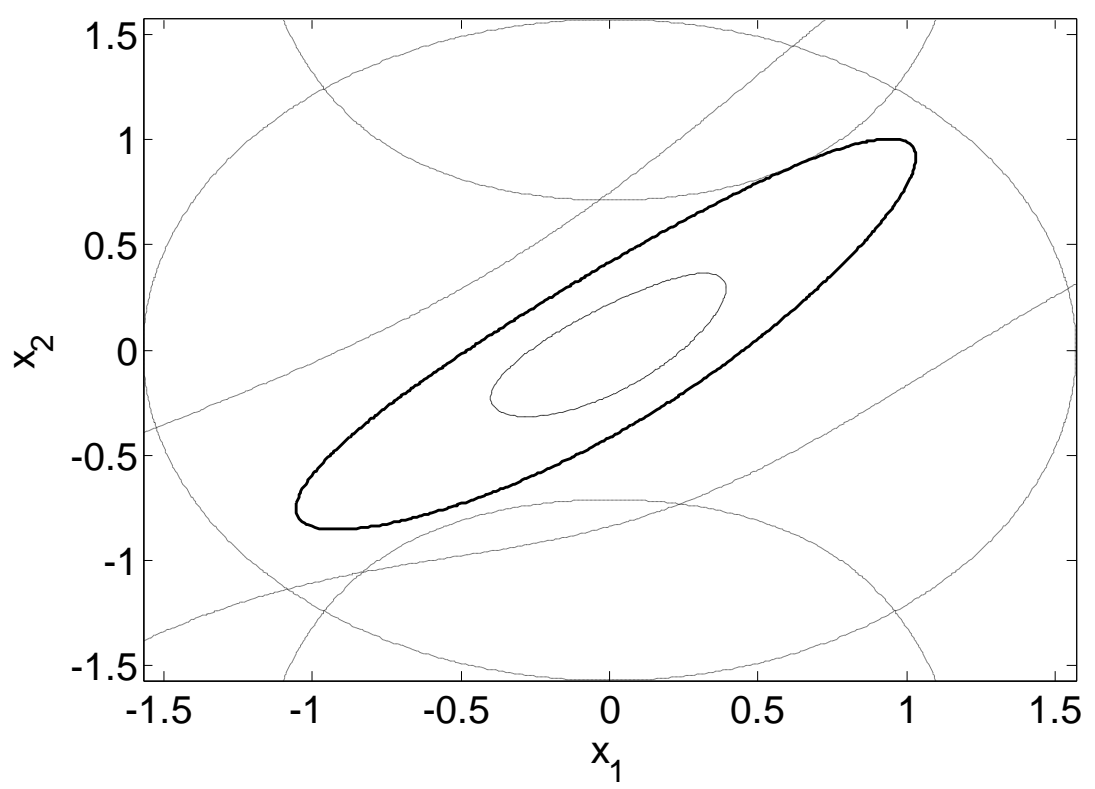

Figure 1: Stabilized domains: with $\mu=30$ (dashed closed curve); with $\mu=100$ (solid closed curve).

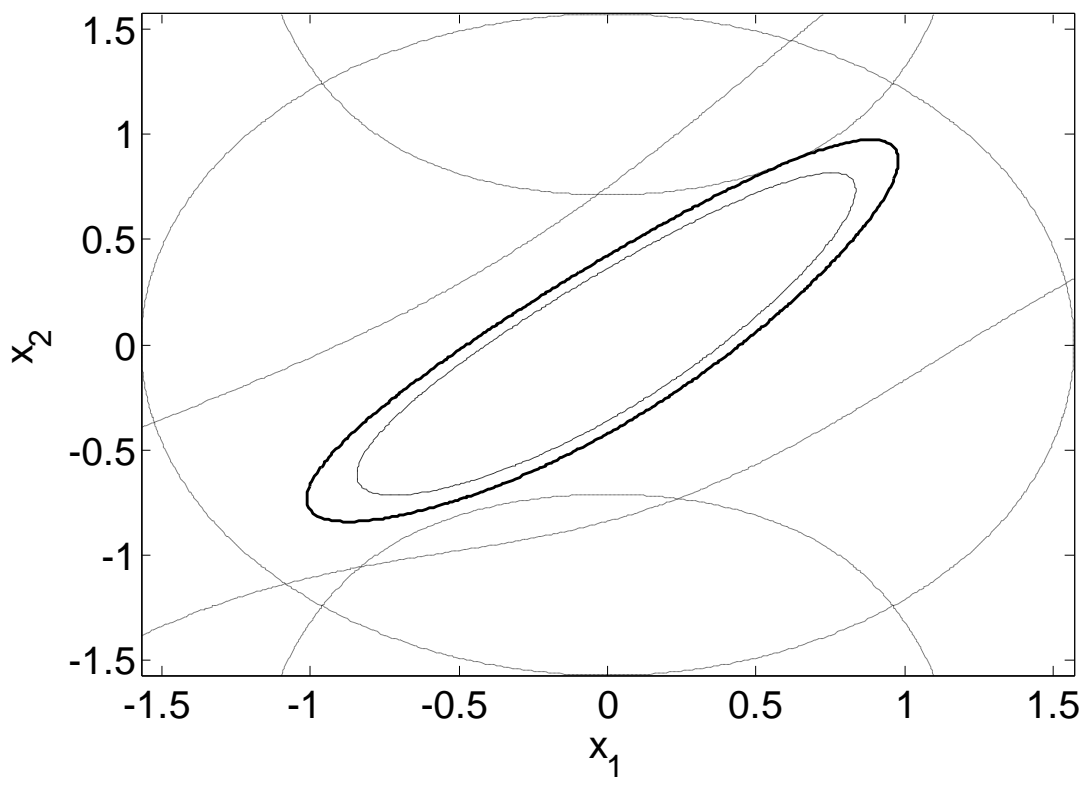

Figure 2: Stabilized domains: without gains $G_{i}^{k}$ (dashed closed curve); with gains $G_{i}^{k}$ (solid closed curve). 
$|u|<20$
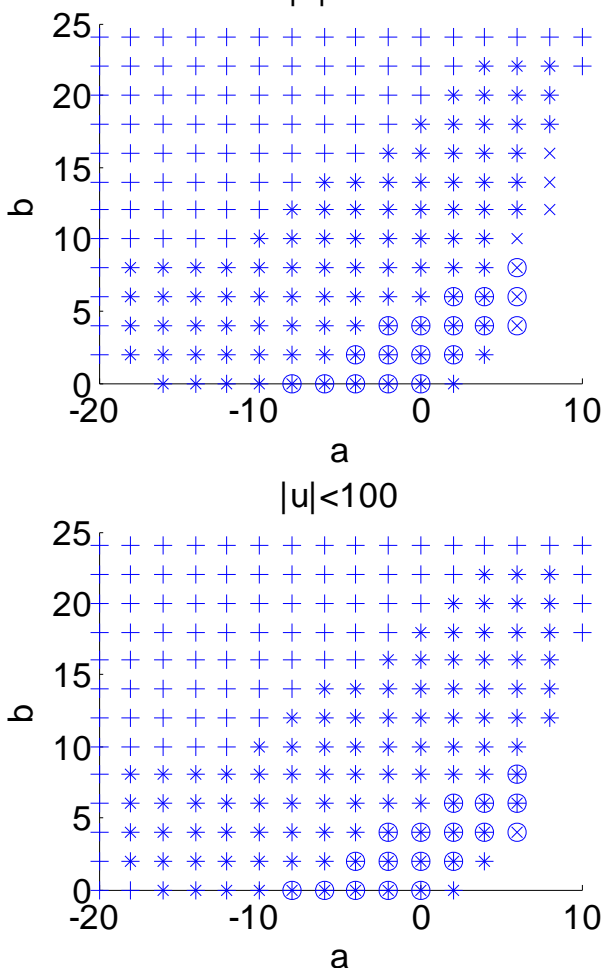

$|u|<30$

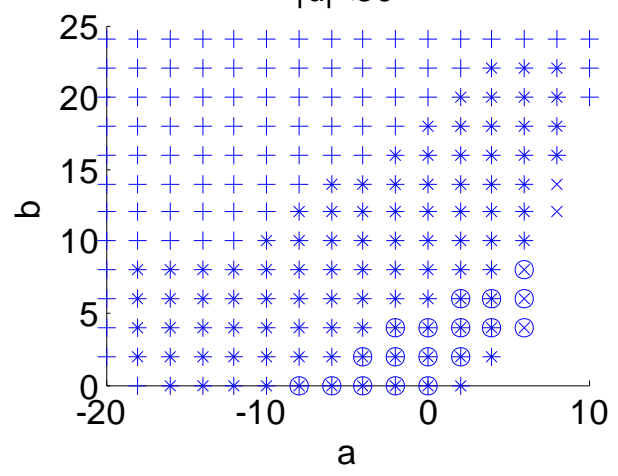

$|\mathrm{u}|<500$

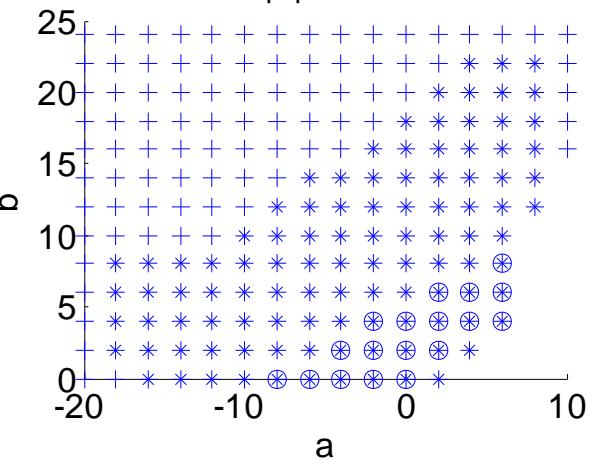

Figure 3: Stabilizability regions (quadratic-“o”, line integral-“x”, proposed approach-“+”).

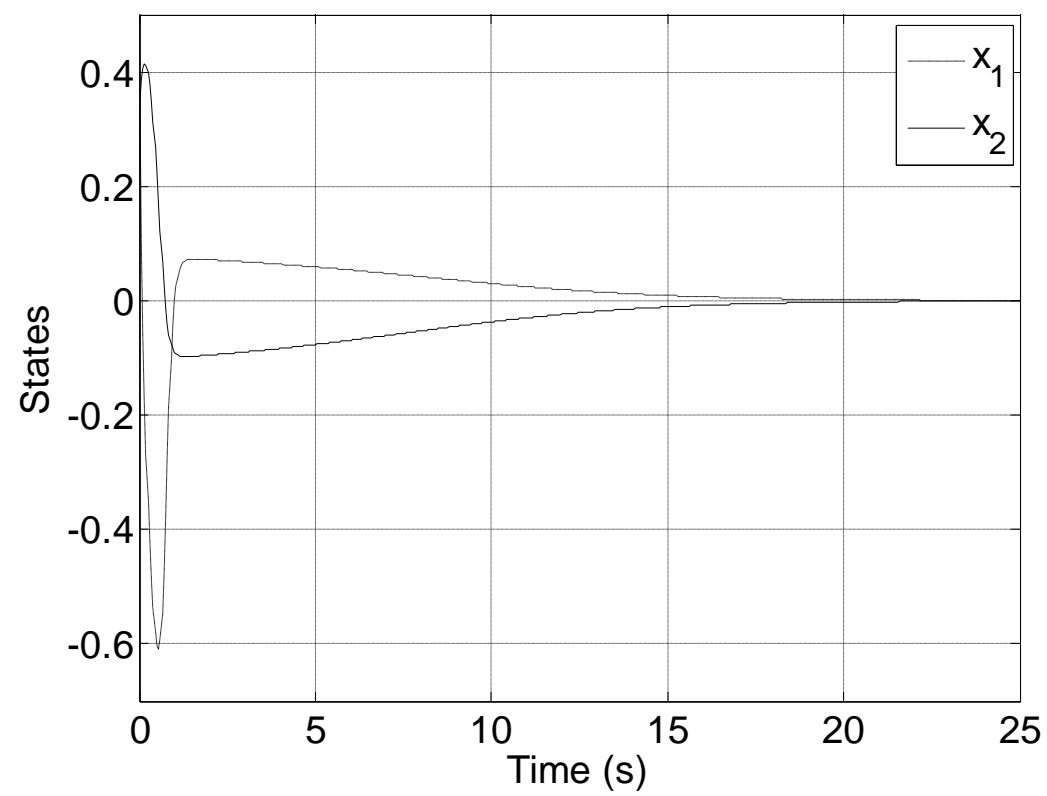

Figure 4: States evolution of TS model (24) under control law (5) obtained via Theorem 3 with $\mu=0.1$,

$$
c=\sqrt{2} \pi / 2 \text {. }
$$




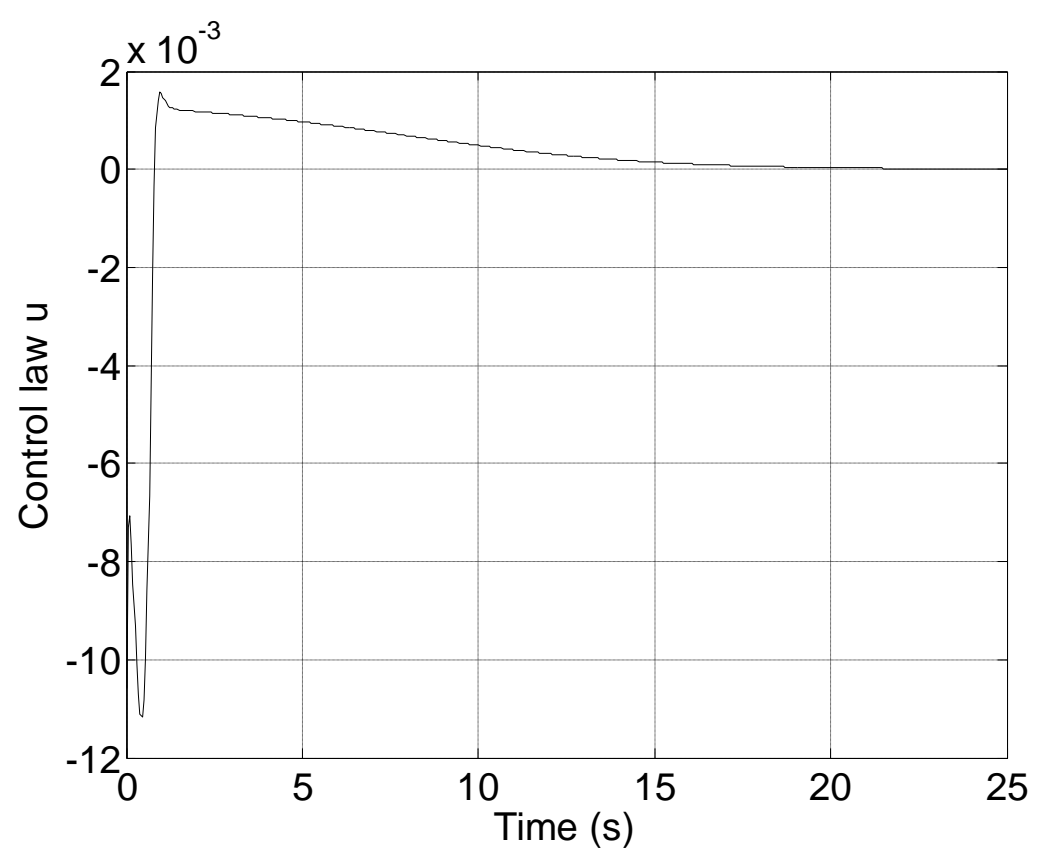

Figure 5: Control law evolution with gains obtained via Theorem 3 with $\mu=0.1, c=\sqrt{2} \pi / 2$.

Table 1: Comparison between Theorems 2 and 3.

\begin{tabular}{|c|c|c|c|c|}
\hline Approach & $\mu=0.01, c=\pi / 2$ & $\mu=0.01, c=\sqrt{2} \pi / 2$ & $\mu=0.1, c=\pi / 2$ & $\mu=0.1, c=\sqrt{2} \pi / 2$ \\
\hline Theorem 2 & $\begin{array}{l}\lambda_{k v s}=0.8836 \\
\eta_{k v}=0.5625\end{array}$ & $\begin{array}{l}\lambda_{k v s}=0.8713 \\
\eta_{k v}=0.5547\end{array}$ & $\begin{array}{l}\lambda_{k v s}=1.4603 \\
\eta_{k v}=0.9297\end{array}$ & $\begin{array}{l}\lambda_{k v s}=1.2149 \\
\eta_{k v}=0.7734\end{array}$ \\
\hline Theorem 3 & $\begin{array}{l}\lambda_{k v s}=0.8836 \\
\eta_{k v}=0.5625\end{array}$ & $\begin{array}{l}\lambda_{k v s}=0.8713 \\
\eta_{k v}=0.5547\end{array}$ & $\begin{array}{l}\lambda_{k v s}=1.4481 \\
\eta_{k v}=0.9219\end{array}$ & $\begin{array}{l}\lambda_{k v s}=1.2026 \\
\eta_{k v}=0.7656\end{array}$ \\
\hline
\end{tabular}

